\title{
Psychiatric problems in fibromyalgia: clinical and neurobiological links between mood disorders and fibromyalgia
}

\author{
A. Alciati ${ }^{1}$, P. Sgiarovello², F. Atzeni ${ }^{2}$, P. Sarzi-Puttini ${ }^{2}$ \\ ${ }^{1}$ Hermanas Hospitalarias, FoRiPsi, Department of Clinical Neurosciences, Villa San Benedetto Menni, \\ Albese con Cassano, Como, Italy; \\ ${ }^{2}$ Rheumatology Unit, L. Sacco University Hospital of Milan, Italy
}

\begin{abstract}
SUMMARY
Objective. To review the literature addressing the relationship between mood disorders and fibromyalgia/chronic pain and our current understanding of overlapping pathophysiological processes and pain and depression circuitry.

Methods. We selectively reviewed articles on the co-occurrence of mood disorders and fibromyalgia/chronic pain published between 1990 and July 2012 in PubMed. Bibliographies and cross references were considered and included when appropriate.

Results. Forty-nine out of 138 publications were retained for review. The vast majority of the studies found an association between depression and fibromyalgia. There is evidence that depression is often accompanied by symptoms of opposite polarity characterised by heights of mood, thinking and behaviour that have a considerable impact on pharmacological treatment. Recent developments support the view that the high rates of fibromyalgia and mood disorder comorbidity is generated by largely overlapping pathophysiological processes in the brain, that provide a neurobiological basis for the bidirectional, mutually exacerbating and disabling relationship between pain and depression.

Conclusions. The finding of comparable pathophysiological characteristics of pain and depression provides a framework for understanding the relationship between the two conditions and sheds some light on neurobiological and therapeutic aspects.
\end{abstract}

Key words: Depression, fibromyalgia, pain, comorbidity.

Reumatismo, 2012; 64 (4): 268-274

\section{INTRODUCTION}

$\mathrm{s}$ more is learned about fibromyalgia (FM), the role of psychosocial factors becomes increasingly recognised to the extent that the condition is now viewed as the result of a complex interaction between biological, psychological and social factors. The growing attention towards the psychiatric disorders associated with FM is also related to consistent findings that unrecognised and untreated psychopathologies can significantly increase pain-related dysfunction and interfere with successful treatment (1). The reciprocally close relationship between FM and psychopathology is further supported by the observation that pain hinders the remission of psychiatric disorders; for example, in the case of major depressive episode, pain delays treatment response (2) and is a significant risk factor for relapse (3).

Previous research has shown that FM is closely associated with a number of psychiatric disorders and psychopathological aspects. In a sample of 115 fifteen females with FM, $77.3 \%$ had psychiatric illnesses diagnosed using the Structured Clinical Interview for DSM-IV (SCID I and II), with $34.8 \%$ having a mood disorder and $32.2 \%$ an anxiety disorder (4). The prevalence of mood and anxiety disorders is more than three times higher in FM patients than in the general population, and match the prevalence rates of other pain illnesses such as rheumatoid arthritis (RA) (5) and chronic back pain (6) in some studies, but not in all (7). The frequency of mood disorders 
in patients with FM has led to consideration of common pathophysiological pathway that may explain the neurobiological mechanism that makes them mutually exacerbating and disabling.

In this review, we first describe a selected sample of current research studies of the association between mood disorders and FM/chronic pain, and then consider investigations into the cerebral pathophysiological pathways that may help to shed some light on the neurobiological basis of the pain/psychopathology interaction.

\section{METHODS}

We searched the literature entered in PubMed from 1990 to July 2012 using the terms "mood disorder," "depression" "major depression," "bipolar disorder," "chronic pain," "fibromyalgia", and "comorbidity." The reference lists of selected articles were reviewed for additional papers of interest that were missed by the computerised database search. Each article was reviewed, and those providing any clinical or pathophysiological information concerning the overlapping of FM/chronic pain and mood disorders were included.

\section{RESULTS}

Three hundred and twelve studies were initially identified as being potentially relevant, of which the full articles of 138 studies were selected and reviewed. Of these, 49 were finally identified as focusing on the relationship between mood disorders and FM/chronic pain, the overlapping pathophysiological processes, and the circuitries of pain and depression.

\section{Mood disorders}

The vast majority of studies and reviews have assessed the role of depression in chronic pain and FM. It has long been known that major depression is highly prevalent in patients with FM, with the rates of current depressive disorders ranging from $28.6 \%$ to $70 \%$, and the life time rates ranging from $62 \%$ to $86 \%(8,9)$, the differences being due to differences in the diagnostic assessment of depression and/ or sampling bias. The close relationship between the two disorders is further supported by the fact that FM is the second most frequent general medical condition associated with major depressive disorder, with an odds ratio (OR) of 3.4 (10).

Depressive symptoms beyond formal diagnosis of major depressive disorder are also frequent in FM patients, with a prevalence of about $40 \%$ as shown in a community study of almost 45,000 subjects (11). An even higher rate has been recently reported by Aguglia (12), who found clinically significant depressive symptoms (as indicated by a Hamilton Rating Scale for Depression (HAM-D) score of $>7$ ) in $83.35 \%$ of the FM patients referred to a Rheumatology Unit.

Over the last few years, converging evidence has been collected that FM patients have high rates of symptoms in the domain of the manic/hypomanic component of bipolar disorder, which are characterised by heights of mood, thinking or activity. Carta (13) detected manic symptoms in 59\% of FM patients, approximately double the rate found in a control sample of healthy subjects. These findings were confirmed by the study of Dell'Osso (14), who found a high rate of lifetime manic symptoms that proved to be related to the severity of pain and its effect on working activities, as well as to a negative impact on overall physical health. In a recent study, we found a high rate $(88 \%)$ of so-called "soft bipolar spectrum disorders" in patients with FM: i.e. disorders in which hypomania or hypomanic symptoms are associated with major or minor depression, or occur in the absence of any depressive condition (15). These findings suggest that hypomanic symptoms (and only secondarily depressive symptoms) may be the core expression of mood disorders in FM. In the light of these observations, the over-active behaviour that has been frequently reported (16) to be characteristic of FM patients can be seen as a manifestation of an hypomanic height of activity (goal-directed over-activity). 
Probably related to the high prevalence of bipolar spectrum disorder, a prospective study of a cohort of 1269 female patients with FM found that the risk of suicide was ten times higher than in the general population (17) and a large-scale community study (the NIMH Epidemiologic Catchment Area Program) has shown that subjects with subsyndromal manic/hypomanic symptoms had a four times higher suicide attempt rate than a comparative group with no mental disorders (18). The high rate of hypomanic symptoms has an important clinical impact given the increasing off-label use of antidepressants to control pain (regardless of the presence of depression) in non-psychiatric settings. The correct detection of the manic/ hypomanic component of mood dysregulation that leads to a diagnosis in the realm of bipolar disorders is crucial, because the treatment of bipolar disorder is different from that of recurrent major depression. Controlled data suggest that there is a risk for inducing mania in patients with a bipolar spectrum disorder who are treated solely with antidepressants (19). Furthermore, their long-term use may have negative consequences such as a poor treatment response (20) or the induction of rapid cycling (21) a severe form of bipolar disorder characterised by four or more episodes of mania or depression in one year. For these reasons, the treatment of bipolar illness classically includes the use of so-called 'mood stabilisers' (lithium and specific anticonvulsants) and atypical antipsychotic agents during both the acute and maintenance phases. Antidepressants should never be used alone, but always together with a mood stabiliser or an atypical antipsychotic agent. Although all of the latter seem to be effective against acute mania, only aripiprazole olanzapine and quetiapine have sufficient data documenting their efficacy in preventing relapse in acute responders (22).

Ninety percent of the studies considered in an evidence-based review (24) concluded that atypical antipsychotic agent have an analgesic effect in pain conditions ranging from headache and cancer pain to chronic lower back pain and FM. Four still-unpub- lished double-blind controlled studies have recently evaluated the efficacy of quetiapine alone or as an add-on in the treatment of FM. Two of these have been presented at congresses, and both suggested that quetiapine could be an effective treatment for FM (25).

A positron emission tomography study of 19 healthy male volunteers provides theoretical support for the clinical view that antipsychotic agents (which share the capacity to antagonise dopamine D2 receptors) may have analgesic properties because it showed that people with relatively few available D2 receptors in the forebrain are likely to have a higher tonic level of pain suppression (26). Moreover, some anticonvulsant agents may improve both bipolar mood disorders and pain. Pregabalin is an anticonvulsant with demonstrated effects on key symptoms of FM such as pain, sleep and fatigue, and has been approved for its management in the United States (27). It has also been observed that pregabalin improves anxiety, a condition that frequently co-occurs with bipolar disorders (28). Valproate and carbamazepine, which are used during both the acute and maintenance phases of bipolar disorder, all have U.S. Food and Drug Administration (FDA) indications for the treatment and/or prophylaxis of some painful conditions (e.g., migraine or trigeminal neuralgia, neuropathic pain and FM) (29).

\section{Effect of mood on pain processing}

The effect of mood on pain processing in FM patients is an important area of study, for both theoretical and practical reasons. A number of findings support the presence of a sensory-discriminative dimension of pain processing that modulates its intensity and spatio-temporal characteristics, and an affective-motivational dimension associated with its negative valence and unpleasantness (30).

The results of various studies converge to suggest that mood selectively alters the affective dimension of pain, as demonstrated by Rainville (31) who showed that pain unpleasantness was more affected by emotions than pain intensity in a group 
of healthy volunteers. A functional magnetic resonance imaging (fMRI) study has endorsed these findings by showing that the extent of depressive symptoms and the presence of comorbid major depression are associated with the magnitude of neuronal activation in the brain regions that process the affective-motivational dimension of pain, and not its sensory-discriminative aspects (32). This finding is supported by some clinical studies, which found that self-rated depressive symptoms, anxiety and catastrophising did not correlate with the ratings of clinical and experimental pain in FM patients. However, depressive symptoms and anxiety can lead to a poor perception of physical health and significantly impair the healthrelated quality of life. $(33,34)$. In a sample of 500 patients with musculoskeletal pain, $54 \%$ reported pain only, $20 \%$ pain and depression, $3 \%$ pain and anxiety, and $23 \%$ pain, depression and anxiety. This last group experienced the greatest pain severity and pain-related disability, with more than twice the number of disability days due to their pain (i.e. 42.6 days in the previous three months $v s$ the 18.1 days of those without concurrent clinical depression or anxiety) (35).

\section{Neural correlates of the relationship be- tween depression and pain}

The relationship between depression and the affective dimension of pain is not surprising because the brain areas involved in regulating mood significantly overlap with those processing the emotional aspects of pain.

The brain system that has been most frequently implicated in the pathophysiology of depression is the extended medial prefrontal network (also called the 'medial network'), a group of ventrally located regions whose core components include the ventromedial prefrontal cortex, and the ventral and posterior regions of the cingulate cortex (36). These brain structures are highly activated during conditions in which healthy subjects attend to their internal emotions or rest passively, a state that frequently focuses on autobiographical thoughts and memories (37), and become deactivated in response to demanding cognitive tasks that require an external focus of attention $(38,39)$.

In agreement with research indicating that effective disengagement from self-referential and negative affective processing is impaired in depressed patients (40), fMRI studies assessing brain responses during emotion processing (41) and cognitive/executive tasks (42) have shown that patients with major depression fail to reduce activity in the medial prefrontal regions, and that this impairment is linked to the severity of their depressive symptoms. Another recent study (43) has shown that, even in response to painful stimuli, patients with major depression fail to deactivate a region involving the subgenual-pregenual anterior cingulate cortex (ACC) and adjacent medial prefrontal areas, with abnormal persistence of activity during pain stimulation.

These findings suggest the presence of increased processing of internal somatic and visceral stimuli in major depressive patients that is consistent with enhanced self-focused attention and a reduction in the resources destined to external stimulation, including painful stimuli. Given the role of the sub-genual ACC in maintaining self-focused attention, the reduced deactivation in this region could explain the paradoxical phenomena of increased somatic complaints in depressive patients and the repeatedly described decreased pain perception during experimentally delivered painful stimulation of the skin (44).

The ACC and the regions including the parietal operculum and posterior insula $(\mathrm{PO} /$ PI) have also been identified as central cortical loci in regulating the emotional and behavioural reaction to pain. (45). It is thought that the ACC is a key structure that contributes to pain unpleasantness, as suggested by human observations showing that surgical ablation of the ACC significantly reduces pain unpleasantness without affecting the ability to detect the intensity or location of pain (46). The role of the ACC is further supported by the observation that specific manipulation of pain unpleasantness leads to significant changes in ACC activity, whereas the manipulation of 
pain intensity mainly leads to changes in the primary somatosensory cortex (31).

The ACC is not only involved in mediating the affective components of pain, but also mediates the anticipation of pain (47). In normal subjects, the uncertain expectation of painful stimulation enhances the brain responses to non-painful warm stimulation in the ACC and PO/PI (i.e. the expectation of painful stimulation amplifies the perceived unpleasantness of even innocuous stimulation), whereas it does not influence perceived pain intensity (48). Increased activation within the amygdale and the ACC during the anticipation of pain has been demonstrated in unmedicated subjects with current depression (49). These inappropriately large responses to anticipated pain suggest an exaggerated emotional distress or affective bias in the pain experience of patients with major depression patients, even before the actual painful stimulation occurs and even though the perception of pain intensity is not altered. This observation suggests that the difference between expected and actual body experiences may be greater in subjects with major depression. This is in line with the assumptions of cognitive models of depression that depressed patients negatively bias their expectations, perceptions and memories, and may represent a neural correlate of their hypervigilant monitoring of negative information (50).

The neuroimaging techniques used to assess the cerebral response to painful stimulation have also been used to investigate the effects of antidepressants. In comparison with untreated control subjects, patients with major depression treated with duloxetine show an improvement in core depressive symptoms that significantly correlates with a significant reduction in fMRI painrelated activations and enhanced deactivations in regions characterised by the abnormal persistence of activity during pain stimulation (34).

Decreased limbic activity also seems to contribute to the effect of cognitive behavioural therapy (CBT), a psychotherapeutic method that has been demonstrated to be effective on various forms of chronic pain (51). CBT is an approach that uses guided discovery to identify and challenge distorted cognitions (i.e catastrophism) and dysfunctional beliefs (52), and maladaptive behavioural patterns such as the avoidance used to prevent pain can be targeted by exposure-oriented interventions. The few studies that have investigated the neural correlates of CBT in pain patients have found that the clinical effect of CBT was associated with significantly decreased resting-state activity in the limbic regions, including the para-hippocampal gyrus and cingulate cortex. It is possible that the decreased limbic activity in response to CBT reflects attenuated vigilance and attention to pain as both of these measures have been found to improve after treatment $(53,54)$.

\section{CONCLUSIONS}

Current evidence supports the idea that depression and FM are highly inter-related, with the co-existence of both being associated with a poor prognosis and greater disability than that observed in patients with either illness alone. Recent findings have shown that depression in patients with FM is often associated with symptoms of opposite polarity, such as the heights of mood, thinking and behaviour characterising the bipolar spectrum of mood disorders. These have an important impact on the pharmacological management of FM and support the use of alternative treatments such as the use anticonvulsant or antipsychotic drugs. Although the underlying mechanism mediating the comorbidity of mood disorder and chronic pain is unknown, there is support for a model in which brain regions (including the limbic and paralimbic prefrontal cortical areas) play a role in both syndromes. These neurobiological links may provide the anatomical basis of the bidirectional relationship between pain and depression, with the symptoms of one worsening the other and/or making the other more likely to develop. Moreover, a better understanding of the relationship between pain and depression may improve current treatment options and provide targets for new treatment strategies. 


\section{REFERENCES}

1. Ohayon MM. Specific characteristics of the pain/depression association in the general population. J Clin Psychiatry. 2004; 65: 5-9.

2. Karp JF, Scott J, Houck P, et al. Pain slows antidepressant treatment response. J Clin Psychiatry. 2005; 66: 591-97.

3. Zis AP, Goodwin FK. Major affective disorder as a recurrent illness: a critical review. Arch Gen Psychiatry. 1979; 36: 835-39.

4. Thieme K, Turk DC, Flor H. Comorbid depression and anxiety in fibromyalgia syndrome: relationship to somatic and psychosocial variables. Psychosom Med. 2004; 66: 837-44.

5. Hawley DJ, Wolfe F. Depression is not more common in rheumatoid arthritis: a 10-year longitudinal study of 6,153 patients with rheumatic disease. J Rheumatol. 1993; 20: 202531.

6. Dickens C, Jayson M, Sutton C, Creed F. The relationship between pain and depression in a trial using paroxetine in sufferers of chronic low back pain. Psychosomatics. 2000; 41: 490-9.

7. Ahles TA, Khan SA, Yunus MB, et al. Psychiatric status of patients with primary fibromyalgia, patients with rheumatoid arthritis, and subjects without pain: a blind comparison of DSM-III diagnoses. Am J Psychiatry. 1991; 148: 1721-6.

8. Gracely RH, Ceko M, Bushnell RC. Fibromyalgia and depression. Pain Res Treat. 2012; 2012: 486590.

9. Arnold LM, Hudson JI, Keck PE, et al. Comorbidity of fibromyalgia and psychiatric disorders. J Clin Psychiatry. 2006; 67: 1219-25.

10. Patten SB, Beck CA, Kassam A, et al. Longterm medical conditions and major depression: strength of association for specific conditions in the general population. Can J Psychiatry. 2005; 50: 195-2.

11. Kato K, Sullivan PF, Evengard B, Pedersen NL. Importance of genetic influences on chronic widespread pain. Arthritis Rheum. 2006; 54: 1682-6.

12. Aguglia A, Salvi V, Maina G, et al. Fibromyalgia syndrome and depressive symptoms: comorbidity and clinical correlates. J Affect Disord. 2011; 128: 262-6.

13. Carta MG, Cardia C, Mannu F, et al. The high frequency of manic symptoms in fibromyalgia does influence the choice of treatment? Clin Pract Epidemol Ment Health. 2006; 2: 36.

14. Dell'Osso L, Bazzichi L, Consoli G, et al. Manic spectrum symptoms are correlated to the severity of pain and the health-related quality of life in patients with fibromyalgia. Clin Exp Rheumatol. 2009; 27: 57-61.

15. Alciati A, Sarzi-Puttini P, Batticciotto A,
Sgiarovello P, Atzeni F, Angst J. Overactive lifestyle in patients with fibromyalgia as a core feature of bipolar spectrum disorder. Ann Rheum Dis 2012 [abstract AB1096].

16. Van Houdenhove B, Neerinckx E, et al. Premorbid "overactive" lifestyle in chronic fatigue syndrome and fibromyalgia. An etiological factor or proof of good citizenship? J Psychosom Res. 2001; 51: 571-6.

17. Dreyer L, Kendall S, Jensen B, et al. Mortality in a Cohort of Danish Patients With Fibromyalgia. Increased Frequency of Suicide. Arthritis Rheum. 2010; 62: 3101-8.

18. Judd LL, Akiskal HS. The prevalence and disability of bipolar spectrum disorders in the US population: re-analysis of the ECA database taking into account subthreshold cases. J Affect Dis. 2003; 73: 123-31.

19. Boerlin HL, Gitlin MJ, Zoellner LA, Hammen CL. Bipolar depression and antidepressant-induced mania: a naturalistic study. J Clin Psychiatry. 1998; 59: 374-9.

20. Ghaemi SN, Hsu DJ, Soldani F, Goodwin FK. Antidepressants in bipolar disorder: the case for caution. Bipolar Disord. 2003; 5: 421-33.

21. Yatham LN, Kennedy SH, O'Donovan C, Parikh SV, Mac Queen G, McIntyre RS et al. Canadian Network for Mood and Anxiety Treatments (CANMAT) guidelines for the management of patients with bipolar disorder: update 2007. Bipolar Disord. 2006; 8: 721-39.

22. Tohen M, Calabrese JR, Sachs GS, et al. Randomized, placebo-controlled trial of olanzapine as maintenance therapy in patients with bipolar I disorder responding to acute treatment with olanzapine. Am J Psychiatry. 2006; 163: 247-56.

23. Thase ME, Macfadden W, Weisler RH, et al. Efficacy of quetiapine monotherapy in bipolar I and II depression: a double-blind, placebocontrolled study (the BOLDER II study). J Clin Psychopharmacol. 2006; 26: 600-9.

24. Fishbain DA, Cutler RB, Lewis J, et al. Do the second-generation "atypical neuroleptics" have analgesic properties? A structured evidence-based review. Pain Med 2004; 5: 359-65.

25. Calandre EP, Rico-Villademoros F. The role of antipsychotics in the management of fibromyalgia. CNS Drugs. 2012; 26: 135-53.

26. Hagelberg N, Martikainen IK, Mansikka H, et al. Dopamine D2 receptor binding in the human brain is associated with the response to painful stimulation and pain modulatory capacity. Pain. 2002; 99: 273-9.

27. Arnold LM, Russell IJ, Diri EW, et al. A 14week, randomized, double-blinded, placebo controlled monotherapy trial of pregabalin in patients with fibromyalgia. J Pain. 2008; 9: 792-5.

28. McIntyre RS, Soczynska JK, Bottas A, et al. 
Anxiety disorders and bipolar disorder: a review. Bipolar Disord. 2006: 8: 665-76.

29. Gill D, Derry S, Wiffen PJ, Moore RA. Valproic acid and sodium valproate for neuropathic pain and fibromyalgia in adults. Cochrane Database Syst Rev. 2011; 10: CD009183.

30. Fernandez E, Turk DC. Sensory and affective components of pain: separation and synthesis. Psychol Bull. 1992; 112: 205-17.

31. Rainville P, Bao QV, Chretien P. Pain-related emotions modulate experimental pain perception and autonomic responses. Pain. 2005; 118: 306-18.

32. Giesecke T, Gracely RH, Williams DA, et al. The relationship between depression, clinical pain, and experimental pain in a chronic pain cohort. Arthritis Rheum. 2005; 52: 1577-4.

33. Jensen KB, Petzke F, Carville S, et al. Anxiety and depressive symptoms in fibromyalgia are related to poor perception of health but not to pain sensitivity or cerebral processing of pain. Arthritis Rheum. 2010; 62: 3488-95.

34. Consoli G, Marazziti D, Ciapparelli A, et al. The impact of mood, anxiety, and sleep disorders on fibromyalgia. Compr Psychiatry. 2012: 486-590.

35. Bair MJ, Wu J, Damush TM, et al. Association of depression and anxiety alone and in combination with chronic musculoskeletal pain in primary care patients. Psychosom Med. 2008; 70: 890-7.

36. Drevets WC, Price JL. Neurocircuitry of mood disorders. Neuropsychopharmacology. 2010; 35: 192-216.

37. Mazoyer B, Zago L, Mellet E, et al. Cortical networks for working memory and executive functions sustain the conscious resting state in man. Brain Res Bull. 2001; 54: 287-98.

38. Harrison BJ, Pujol J, Lopez-Sola M, et al. Consistency and functional specialization in the default mode brain network. P Natl Acad Sci USA. 2008; 105: 9781-6.

39. Raichle ME, MacLeod AM, Snyder AZ, et al. A default mode of brain function. P Natl Acad Sci USA. 2001; 98: 676-82.

40. Siegle GJ, Steinhauer SR, Thase ME, et al. Can't shake that feeling: event-related fMRI assessment of sustained amygdala activity in response to emotional information in depressed individuals. Biol Psychiatry. 2002; 51: 693-7.

41. Grimm S, Boesiger P, Beck J, et al. Altered negative BOLD responses in the default-mode network during emotion processing in depressed subjects. Neuropsychopharmacology. 2009; 34: 932-43.

42. Vasic N, Walter H, Sambataro F, Wolf RC. Aberrant functional connectivity of dorsolat- eral prefrontal and cingulate networks in patients with major depression during working memory processing. Psychol Med. 2009; 39: 977-87.

43. López-Solà M, Pujol J, Hernández-Ribas R, et al. Effects of duloxetine treatment on brain response to painful stimulation in major depressive disorder. Neuropsychopharmacology. 2010; 35: 2305-7.

44. Dickens C, McGowan L, Dale S. Impact of depression on experimental pain perception: a systematic review of the literature with meta-analysis. Psychosom Med. 2003; 65: 369-75.

45. Becerra LR, Breiter HC, Stojanovic M, et al. Human brain activation under controlled thermal stimulation and habituation to noxious heat: an fMRI study. Magn Reson Med. 1999; 41: 1044-57.

46. Hurt RW, Ballantine HTJ. Stereotactic anterior cingulate lesions for persistent pain: a report on 68 cases. Clin Neurosurg. 1974; 21 : 334-51.

47. Ploghaus A, Tracey I, Gati JS, et al. Dissociating pain from its anticipation in the human brain. Science. 1999; 284: 1979-81.

48. Sawamoto N, Honda M, Okada T, et al. Expectation of pain enhances responses to nonpainful somatosensory stimulation in the anterior cingulate cortex and parietal operculum/ posterior insula: an event-related functional magnetic resonance imaging study. J Neurosci. 2000; 20: 7438-45.

49. Strigo IA, Simmons AN, Matthews SC, et al. Association of major depressive disorder with altered functional brain response during anticipation and processing of heat pain. Arch Gen Psychiatry. 2008; 65: 1275-84.

50. Whalen PJ, Shin LM, Somerville LH, et al. Functional neuroimaging studies of the amygdala in depression. Semin Clin Neuropsychiatry. 2002; 7: 234-42.

51. Bernardy K, Füber N, Köllner V, Häuser W. Efficacy of cognitive-behavioral therapies in fibromyalgia syndrome - a systematic review and metaanalysis of randomized controlled trials. J Rheumatol. 2010; 37: 1991-2005.

52. Beck A. Cognitive models of depression. J Cogn Psychother. 1987; 1: 5-37.

53. Lackner JM, Lou Coad M, Mertz HR, et al. Cognitive therapy for irritable bowel syndrome is associated with reduced limbic activity, GI symptoms, and anxiety. Behav Res Ther. 2006; 44: 621-38.

54. Jensen KB, Kosek E, Wicksell R, et al. Cognitive behavioral therapy increases pain-evoked activation of the prefrontal cortex in patients with fibromyalgia. Pain 2012; 153: 1495-03. 